\title{
Changes in Pitch with a Cochlear Implant Over Time
}

\author{
Lina A. J. Reiss, ${ }^{1}$ Christopher W. Turner, ${ }^{1,2}$ Sheryl R. Erenberg, ${ }^{1,2}$ and Bruce J. Gantz ${ }^{2}$ \\ ${ }^{1}$ Department of Speech Pathology and Audiology, Wendell Johnson Speech and Hearing Center, University of Iowa, \\ Iowa City, IA 52242, USA \\ ${ }^{2}$ Department of Otolaryngology-Head Neck Surgery, University of Iowa, Iowa City, IA 52242, USA
}

Received: 7 September 2006; Accepted: 26 January 2007; Online publication: 9 March 2007

\begin{abstract}
In the normal auditory system, the perceived pitch of a tone is closely linked to the cochlear place of vibration. It has generally been assumed that highrate electrical stimulation by a cochlear implant electrode also evokes a pitch sensation corresponding to the electrode's cochlear place ("place" code) and stimulation rate ("temporal" code). However, other factors may affect electric pitch sensation, such as a substantial loss of nearby nerve fibers or even higher-level perceptual changes due to experience. The goals of this study were to measure electric pitch sensations in hybrid (short-electrode) cochlear implant patients and to examine which factors might contribute to the perceived pitch. To look at effects of experience, electric pitch sensations were compared with acoustic tone references presented to the non-implanted ear at various stages of implant use, ranging from hookup to 5 years. Here, we show that electric pitch perception often shifts in frequency, sometimes by as much as two octaves, during the first few years of implant use. Additional pitch measurements in more recently implanted patients at shorter time intervals up to 1 year of implant use suggest two likely contributions to these observed pitch shifts: intersession variability (up to one octave) and slow, systematic changes over time. We also found that the early pitch sensations for a constant electrode location can vary greatly across subjects and that these variations are strongly correlated with speech reception performance. Specifically, patients with an early
\end{abstract}

Correspondence to: Lina A. J. Reiss • Department of Speech Pathology and Audiology, Wendell Johnson Speech and Hearing Center • University of Iowa - Iowa City, IA 52242, USA. Telephone: +1-3193358718; fax: +1-319-3358851; email: Lina-Reiss@uiowa.edu low-pitch sensation tend to perform poorly with the implant compared to those with an early high-pitch sensation, which may be linked to less nerve survival in the basal end of the cochlea in the low-pitch patients. In contrast, late pitch sensations show no correlation with speech perception. These results together suggest that early pitch sensations may more closely reflect peripheral innervation patterns, while later pitch sensations may reflect higher-level, experience-dependent changes. These pitch shifts over time not only raise questions for strict place-based theories of pitch perception, but also imply that experience may have a greater influence on cochlear implant perception than previously thought.

Keywords: cochlear implant, hybrid, frequency, tonotopy, speech, plasticity

\section{INTRODUCTION}

The cochlear implant is a surgical intervention for severe hearing loss that uses an electrical device to stimulate the auditory nerve directly, bypassing the receptor cell pathway, which has been damaged as a result of hearing loss. It is a highly successful strategy for treating deafness, even though it is not yet fully clear how this device works in conjunction with the human brain. In the normal auditory system, basilar membrane frequency tuning is tonotopic, systematically increasing from one end (apex) to the opposite end (base); this tonotopicity is preserved in the hair cells and associated nerve fibers up to and including the auditory cortex. To approximate frequency selectivity and tonotopicity with a cochlear implant, each electrode in the implant is positioned at a different location in the cochlea. 
Correct allocation of acoustic frequencies to individual electrodes may be important for music and speech recognition (Dorman et al. 1997; Fu and Shannon 1999a,b; Faulkner et al. 2003; Baskent and Shannon 2004), although some studies suggest that implant users can adapt over time to spectrally shifted speech-frequency mappings (Rosen et al. 1999; Fu et al. 2005).

Previous pitch-ranking studies in traditional cochlear implant users have indicated that both electrode location (place pitch cues) and stimulation rate (temporal pitch cues) can influence the perceived pitch (e.g., Tong et al. 1982; Shannon 1983; Townshend et al. 1987; McKay et al. 2000). For instance, a more basal electrode will elicit a higher pitch than a more apical one, and a higher stimulation rate will elicit a higher pitch than a lower rate, up to around $300 \mathrm{~Hz}$ where the rate pitch cue saturates. However, the absolute relationship of these parameters to pitch, especially electrode location (place pitch), is unclear from this literature. Is the perceived frequency determined by the dendritic nerve fibers in that region of the cochlea, or does the electrical current travel farther to stimulate axons or spiral ganglion cells in the modiolus? What if there are only sparse areas of surviving nerve? Finally, how does the central auditory system respond to this change in input, i.e., the potentially very different patterns of stimulation by the implant, and does this have any influence on pitch perception?

A previous major obstacle to addressing this question was that most cochlear implant patients had almost no residual hearing in the opposite ear for an objective comparison of pitch. However, in recent years, due to the increasing success rates of cochlear implants, implantation criteria have been relaxed to allow implantation of patients with less severe hearing loss. There have been a few studies of absolute implant pitch perception, with generally mixed results. Three studies comparing implant pitch sensations to acoustic tone references have found pitch sensations to be variable, ranging from one to three octaves lower than expected from electrode location on the basilar membrane (Blamey et al. 1996b; Dorman et al. 1994; Boex et al. 2006). Another study that looked indirectly at pitch sensations through contralateral masking patterns also found pitch sensations to be mainly lower than predicted (James et al. 2001). Just one other study found implant pitch sensations to be generally consistent with cochlear location (Eddington et al. 1978).

Recently, a new type of implant, the Iowa/Nucleus hybrid implant (Gantz and Turner 2003; Turner et al. 2004), has been designed specifically for those with significantly more residual hearing than the typical implant candidate. This hybrid (short electrode) cochlear implant is a shorter, thinner version of the traditional cochlear implant and is implanted only into the basal, or high frequency, region of the cochlea without directly affecting usable low-frequency acoustic hearing. These patients also typically have considerable residual hearing in the opposite, unimplanted ear and can often match the pitch sensations between the two ears to frequencies as high as $2-4 \mathrm{kHz}$.

Furthermore, as the hybrid implant is only implanted into the basal region of the cochlea, nerve survival in this region may be a predictive factor for speech reception performance. Previous studies of traditional cochlear implants have not found correlations between nerve survival and speech perception (Nadol et al. 2001; Khan et al. 2005), but the hybrid implant may differ in that it is more localized, and local nerve survival may be relatively more important for this type of implant than other factors. Specifically, greater local nerve survival in the cochlear base would presumably mean more discrete stimulation of nearby neurons by individual electrodes, leading to better speech discrimination performance. Conversely, less nerve survival in the base would mean broad and nonspecific stimulation of neurons further away toward the apex, leading to poorer speech discrimination performance. Pitch may be useful as an indirect indicator of nerve survival, i.e., high pitch sensations may indicate basal nerve survival and low pitch sensations may indicate no basal nerve survival.

To investigate the effects of experience on electric pitch perception, we studied pitch perception in adult hybrid cochlear implant subjects over various durations of implant use ranging from initial device hookup to 5 years. We found that perceived pitch from the most apical electrode can shift over long time periods of a year or more and by more than two octaves in some patients. We also investigated whether there is a relationship between pitch perception and speech understanding in hybrid implant users. The results suggest a relatively strong predictive value for early pitch sensations measured before 1 year of implant experience, but not late pitch sensations.

\section{METHODS}

\section{Subjects}

These studies were conducted according to the guidelines for the protection of human subjects as set forth by the Institutional Review Board (IRB) of the University of Iowa, and the methods employed were approved by that IRB.

Eighteen adult hybrid cochlear implant subjects, with ages ranging from 19 to 75 at age of implantation, participated in this study. As described in the introduction, the hybrid implant is a shorter, thinner 
version of the traditional cochlear implant and is implanted only into the basal, or high frequency, region of the cochlea. The goal is to provide electric hearing to regions of profound hearing loss without affecting residual low-frequency hearing (Gantz and Turner 2003; Turner et al. 2004). Because of the shorter length, the most apical electrode in the hybrid implant is implanted approximately $10.5 \mathrm{~mm}$ from the cochlear base. There are six active electrodes in the hybrid version (compared with 22 active electrodes for the regular Nucleus 24 or Freedom), each spaced about $0.8 \mathrm{~mm}$ apart. Postoperative x-rays in several subjects demonstrate a smooth banana-shaped gentle curve to the electrode in the base of the cochlea. It is unlikely that there have been any electrode shifts, as the speech perception scores continue to improve in most over time. The relative simplicity of this insertion (straight insertion into the basal region with small variance of angle) suggests less variability in insertion depth compared to traditional electrodes that must negotiate cochlear turns. When potential variability due to angle or cochlear length is accounted for, the Greenwood frequency-place function for the basilar membrane predicts a pitch sensation between 2,800 and $4,700 \mathrm{~Hz}$ for the most apical electrode (Greenwood 1990; Leake et al. 2006). The remaining electrodes would have correspondingly higher frequencies.

Of these 18 subjects, pitch data were collected for 12 subjects over various time points during implant use, and for the remaining six subjects, over only one time point. The latter subjects were only sampled once because they were from out of town (other clinical centers) or could not pitch match within their residual frequency range at other time points. Note that while the first group's pitch data are shown as a function of time in Figures 2, 4, and 5, the second group's pitch data are only included (along with the first group's data) in the correlations of speech with pitch, as described below.

Speech perception was tested using subjects' everyday MAP frequency allocations (table of speech processor frequency allocations for each electrode) and signal processing strategies [all used an advanced combinational encoder (ACE) strategy with rates ranging from 720 to 2,400 pps; five channels in two subjects where an electrode was deactivated; six channels in all other subjects]. The hybrid subjects' ages, durations of implant use, ipsilateral low-frequency hearing loss (all have a profound loss at frequencies above $1,000 \mathrm{~Hz}$ ), ipsilateral hearing aid use, processor type, MAP frequency range, and best speech reception scores (speech materials described in Speech reception testing section below) are shown in Table 1. Most subjects had a gradual onset of hearing loss with unknown etiology; therefore, duration of hearing loss and etiology were not analyzed.
Note in particular the relatively low MAP frequency allocation ranges compared to the actual implant intracochlear location. Hybrid implant patients typically prefer a MAP with a lower and broader frequency allocation by the speech processor. If given a choice of total frequency allocation ranges of $600-8,000 \mathrm{~Hz}$, $1,500-8,000 \mathrm{~Hz}$, or $3,000-8,000 \mathrm{~Hz}$ (to be divided among six electrodes), most will choose the lowest and broadest frequency range, most likely because the widest range of frequencies provides the greatest speech information.

\section{Electric to acoustic pitch matches}

Clinical or research software from Cochlear (R126/ Custom Sound or NIC) was used to stimulate a single electrode in the hybrid implant, most often electrode 6 or the most apical electrode in the implant. When there was time and a sufficient audible frequency range in the contralateral ear to match higher frequencies, the more basal electrodes 4 and 2 were also tested. The electrode ground was always monopolar (MP1+2). Electrical stimuli were presented at C-level or "loud but comfortable" current level. A high pulse rate was always used to "saturate" temporal effects on pitch perception, i.e., rates of at least 800 pulses per second (pps), usually 1,000 or 1,200 pps, were used so that any pulse rate effects would be invariant. No significant changes in pitch perception were observed with variations in current level or pulse rates above 800 pps (Fig. 7; see results).

Sennheiser HD-25 headphones were used to play acoustic tones to the non-implanted ear. Depending on method, either an analog tone generator/attenuator or Creative Labs EMU 1818M sound card/TDT PA4 digital attenuator was used to generate and play the acoustic tones.

Two methods, one manual and one computerized, were used to match electric pitches to acoustic tone references. Both methods gave similar results and are described below.

Generally, electric stimuli were presented first, followed by an interstimulus interval delay, then acoustic tone stimuli. Subjects were instructed to rate whether the acoustic tone was higher, lower, or similar in pitch, while ignoring loudness (loudness roving was not used because maximum audibility, and thus, maximum sound level was desired in the frequency range of greatest hearing loss, often at the highest frequencies). Acoustic tone frequency was varied until the range of similar pitches was bracketed. If a patient was unable to bracket the perceived pitch with acoustic tones presented to the non-implanted ear (usually due to an inability, due to severe hearing loss, to hear acoustic tones higher in pitch than the electric stimulation), then no pitch match data were recorded 


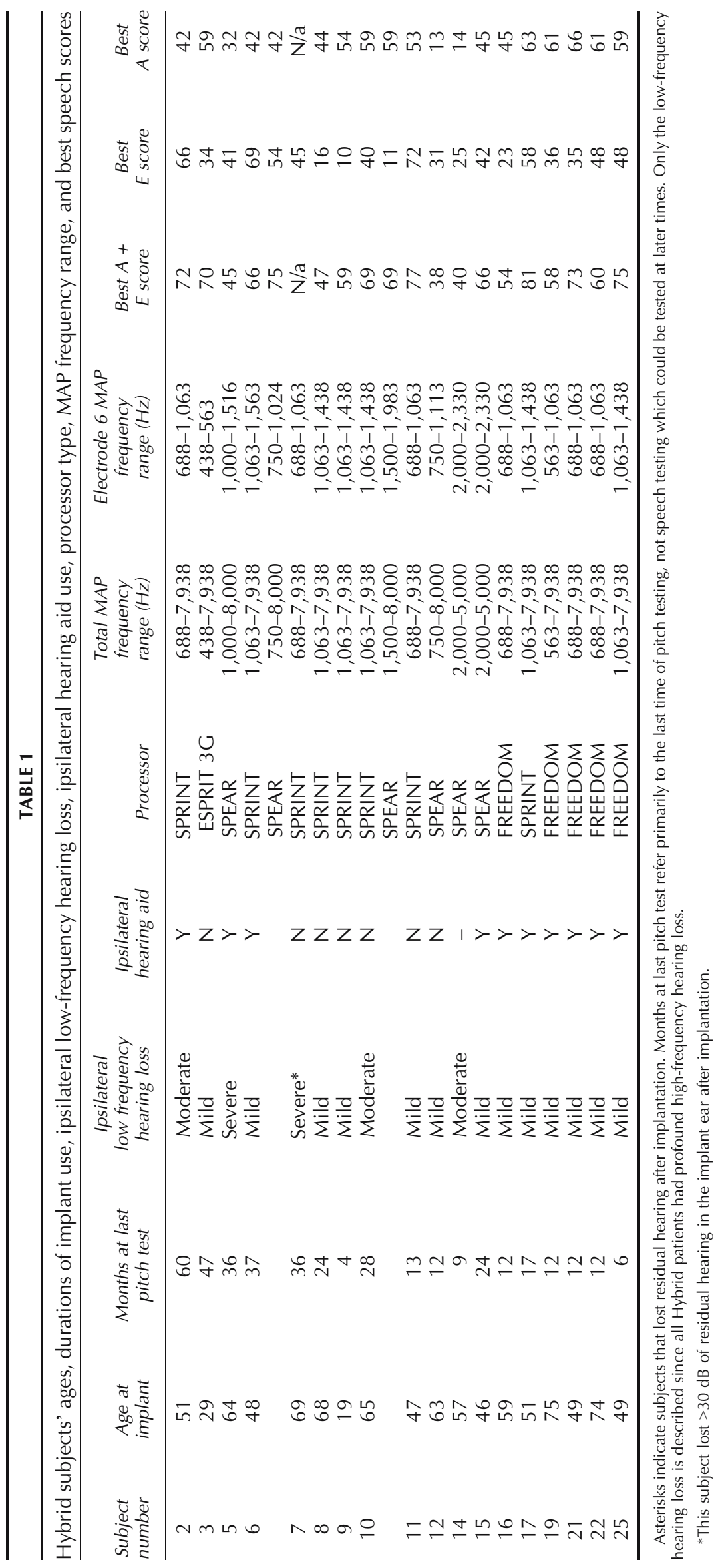


a

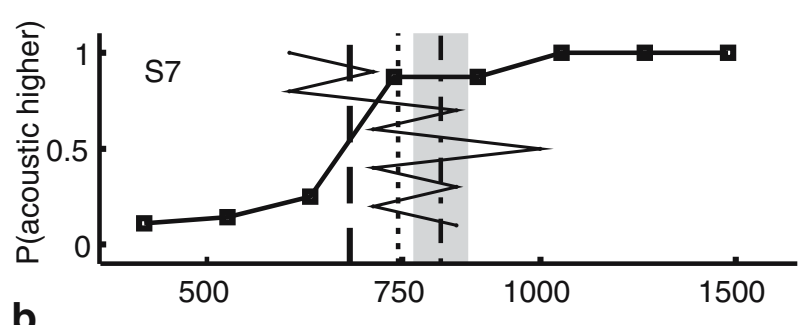

b

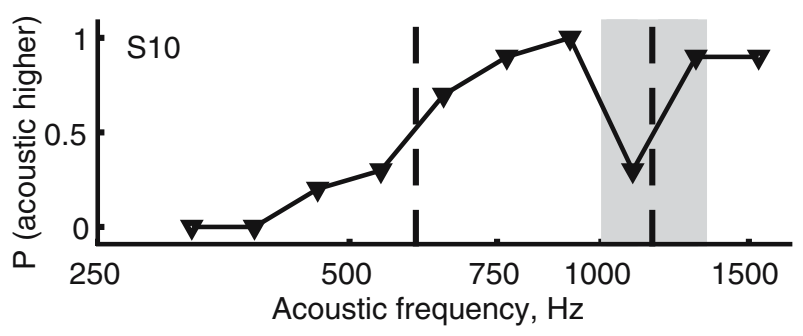

FIG. 1. (a) Example results for a single subject (S7) with three different pitch estimation methods. Heavy line, open squares: psychometric function collected with eight to ten repetitions of acoustic pitch comparisons at each frequency. Heavy dashed vertical line indicates $50 \%$ point of function. Thin solid jagged line: path of reversals for up-down pitch matching task. Middle dotted vertical line indicates average of the reversals. Gray shaded area indicates range of acoustic frequencies considered similar in manual matching paradigm. Dash-dot line indicates the middle of this range. (b) Example of unusual non-monotonic psychometric function collected for subject S10. The heavy dotted lines indicate the two $50 \%$ points obtained from this function. The higher value coincides with the middle of the range obtained from the manual paradigm (gray shaded region).

for that session. If there was doubt about the patient's ability to pitch match, or otherwise ignore loudness effects, they were tested on "practice" runs of pitch ranking of different acoustic tones presented to the contralateral ear. Data were not included if the patient was unable to perform acoustic-acoustic pitch ranking, or if the patient's responses were highly inconsistent (e.g., responses did not vary monotonically from "acoustic lower" to "same" to "acoustic higher" with increasing frequency, or responses were not consistent within one octave when a tone was repeated) within a single-pitch matching session.

In the manual method, three electric pulse trains of $500 \mathrm{~ms}$ duration and interstimulus interval were presented via Cochlear clinical software (R126 or Custom Sound), and then three acoustic pulsed pure-tones were presented immediately with the tone generator at an audible sound level. Acoustic tones were randomly selected by the experimenter within a decreasing frequency range to bracket the matching pitch range.

In the computerized method, acoustic tones were first loudness matched at all audible frequencies in one-octave steps to electric pulses using a threealternative (lower, higher, or same), up-down protocol, first two runs in $10 \mathrm{~dB}$ and then six runs in 4-dB steps. The loudness-matched sound level was taken to be the average of the reversals for the 4-dB steps. For pitch matching, one electric pulse train of 400-ms duration (generated with the NIC software) and one acoustic tone of 400-ms duration (generated with the sound card and digital attenuator) were presented alternately with a 400-ms interstimulus interval. The stimulus order was randomized, with either the electric or acoustic stimulus occurring first. Acoustic tones were selected either to converge in a twoalternative forced choice up-down task (Levitt 1971) or a Latin squares pseudorandom sequence (to minimize stimulus sequence effects on pitch comparisons). In both tasks, subjects were instructed to indicate which stimulus was higher in pitch. In the up-down task, the first 4 runs were in one-octave steps, and the last 12 runs were in one-fourth-octave steps. In the randomized task, a Latin-square sequence of audible frequencies in one-octave steps were presented to estimate a two-octave range of pitch match, and then similarly, frequencies in onefourth octave steps over this two-octave range were presented to narrow down the pitch match. The pitch matches were computed as the average of the last six reversals in the up-down task or the $50 \%$ point in the psychometric function for one-fourthoctave step frequencies in the randomized task.

These two methods often gave similar results in pitch matches (within the error seen in a single day), as shown in Figure 1a. Generally, the manual method was used to collect the majority of the initial data points (shown as circles in Figs. 2, 4, and 5), due to both time constraints and the greater flexibility and utility of feedback from subjects. The computerized methods were used to collect additional data points and to verify that pitch matches obtained manually were independent of loudness cues; these data are typically represented when multiple data points for a single time are shown (shown in Figs. 2, 4, 5, and 7 as triangles and squares for up-down and random methods, respectively).

\section{Speech reception testing}

To determine how pitch sensations relate to performance with the implant, pitch sensations were compared with speech reception performance. Subjects were tested on discrimination of 16 consonants presented in a /a/-consonant-/a/ context (Turner et al. 1995). Each consonant was spoken by four different talkers. Performance was tested under the electric-alone condition and under the acoustic + electric and acoustic-alone conditions, if there was 

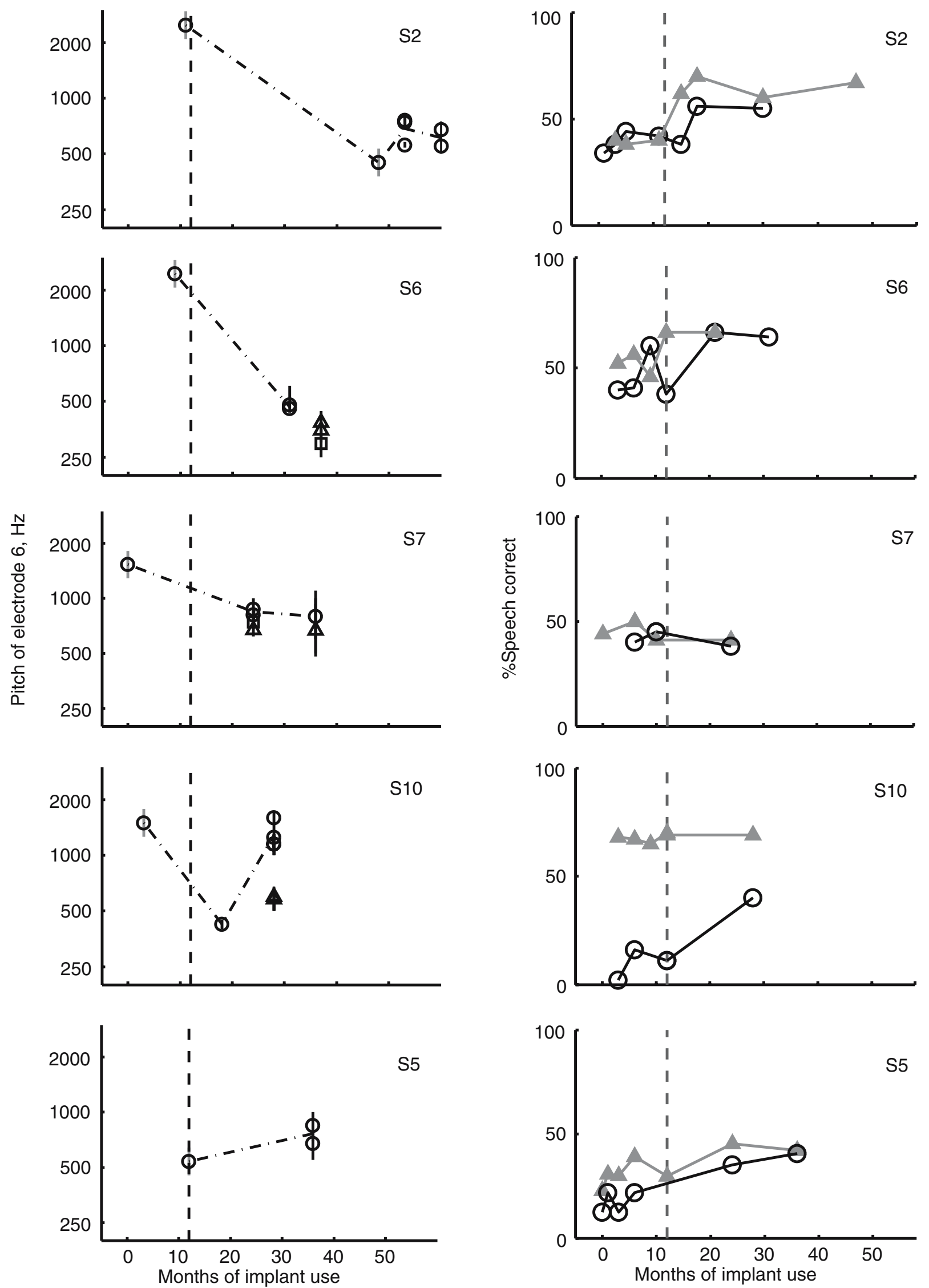
time. The latter two conditions were conducted via loudspeaker with the non-implant ear plugged, while stimuli in the electric-only condition were presented via a direct electric connection. Speech scores under the electric-only condition were comparable with speech scores under loudspeaker with both ears plugged and muffed, but not ear plugs alone (ear plugs provide $<30 \mathrm{~dB}$ of attenuation, not sufficient for subjects with mild low-frequency hearing losses). All experiments were conducted in a sound-attenuating booth.

Speech reception was tested at various times along with the pitch match data; some of these data are shown in Figure 2b. However, to determine if pitch predicts long-term speech scores, scores were compared with both early and late pitch sensations only after speech reception performance had reached long-term asymptotic levels ( $>7$ months after implant hookup).

\section{RESULTS}

\section{Acoustic to electric pitch matches}

Five subjects had multiple pitch matches for the most apical electrode over very long time periods ranging from 2-5 years of implant use. These pitch matches over time are shown in the left column of Figure 2 as the linear center of the pitch-matched range (range of frequencies matched to the electric pitch as "similar", e.g., gray shaded area in Fig. 1a). The entire pitch-matched range (range of frequencies considered "similar") for each data point is also shown as vertical lines around each data point; these ranges or brackets are generally on the order of onehalf- to one-octave wide (e.g., Fig. 1a,b; Fig. 7). Note that these vertical lines represent bracketed pitch match ranges, not standard deviations (variance). The variance of the data is indicated instead by the scatter of individually plotted repeat estimates, at times when repeat estimates were taken.

These results show that the absolute pitch sensations for three out of five of these subjects (S2, S6, and S7) clearly dropped in frequency over time and by more than two octaves for two of these subjects. The fourth subject (S10) was slightly different from the others in that there was a bimodal pitch match to two different frequency regions at the latest time point, apparent in the two groups of pitch data points at 28 months in the left column of Figure 2; this is further illustrated by the pitch psychometric function for this same subject shown in Figure 1b. In other words, the psychometric function shows that there were, in fact, two frequency regions where the subject was $50 \%$ likely to say that the acoustic pitch was higher, which suggests that there were two frequency regions heard as similar in pitch to the electric pitch (in Fig. 2, only the lower pitch estimate from this method is plotted for clarity). The lower pitch would suggest a drop in this subject's perceived pitch over time, but the presence of the higher pitch suggests no change. The presence of bimodal pitches also suggests a possibility that this subject may be undergoing a sudden, rather than, gradual transition in pitch sensations, i.e., the subject may be matching both the "old" and "new" pitch sensation.

The fifth subject (S5) showed a slight increase in pitch over time. Note that this subject differed from the others in that the earliest pitch sensation recorded at 12 months was a relatively low rather than high pitch.

While some variability over short time scales is evident for all subjects, this variability is within the range of error seen in a single day (as large as one-half to one octave). The changes in pitch sensation seen in two of the subjects over the long time scales shown are much larger than this variability over short time scales and the one-half- to one-octave brackets typically observed. From the available data for these subjects, it is unclear whether these pitch changes occur gradually over a long time scale (some continuous function) or suddenly at some unknown time between measurements (a step function); hence, the dashed-dot lines.

Also shown for comparison are these same subjects' speech perception scores over time (Fig. 2, right column) and audiograms over time (Fig. 3). The right column of Figure 2 shows that the speech perception scores either remained the same or improved slightly over the same time period as the changes in pitch sensation, suggesting that speech

FIG. 2. Pitch sensations and speech performance of five individual subjects plotted over long time scales ( $>2$ years). Left column: mean values of pitch matches of the most apical electrode (electrode 6 ) tended to change after 12 or more months of implant use (indicated by vertical dotted line), sometimes by more than two octaves. Different symbols indicate different methods of pitch matching: manual (circles), pseudorandomized (triangles), or up-down task (squares). The dot-dashed lines indicate the averages of the manually estimated pitch matches over time. The entire pitch-matched range (range of frequencies considered "similar") for each data point is also shown as solid vertical lines around each data point; except for some early data points where the "similar range" is estimated as $1 / 2$ octaves wide and plotted as thick gray vertical lines around each data point. A small amount of variability over short time scales is evident for subjects S2 and S6; this variability is within the range of error seen in a single day (as large as 1/2-1 octave), and much smaller than the change seen over long time scales. Subject S10, however, has a large variability at the last measurement, which is explained by the presence of two regions of pitch match psychometric function from the random method (see Fig. 1b). Right column: speech scores over time for the same subjects. Both acoustic + electric combined scores (gray filled triangles) and electric-only scores (black open circles) are shown over time. Speech scores either did not change or improved slightly over the same time period of the pitch change. 
Ipsilateral
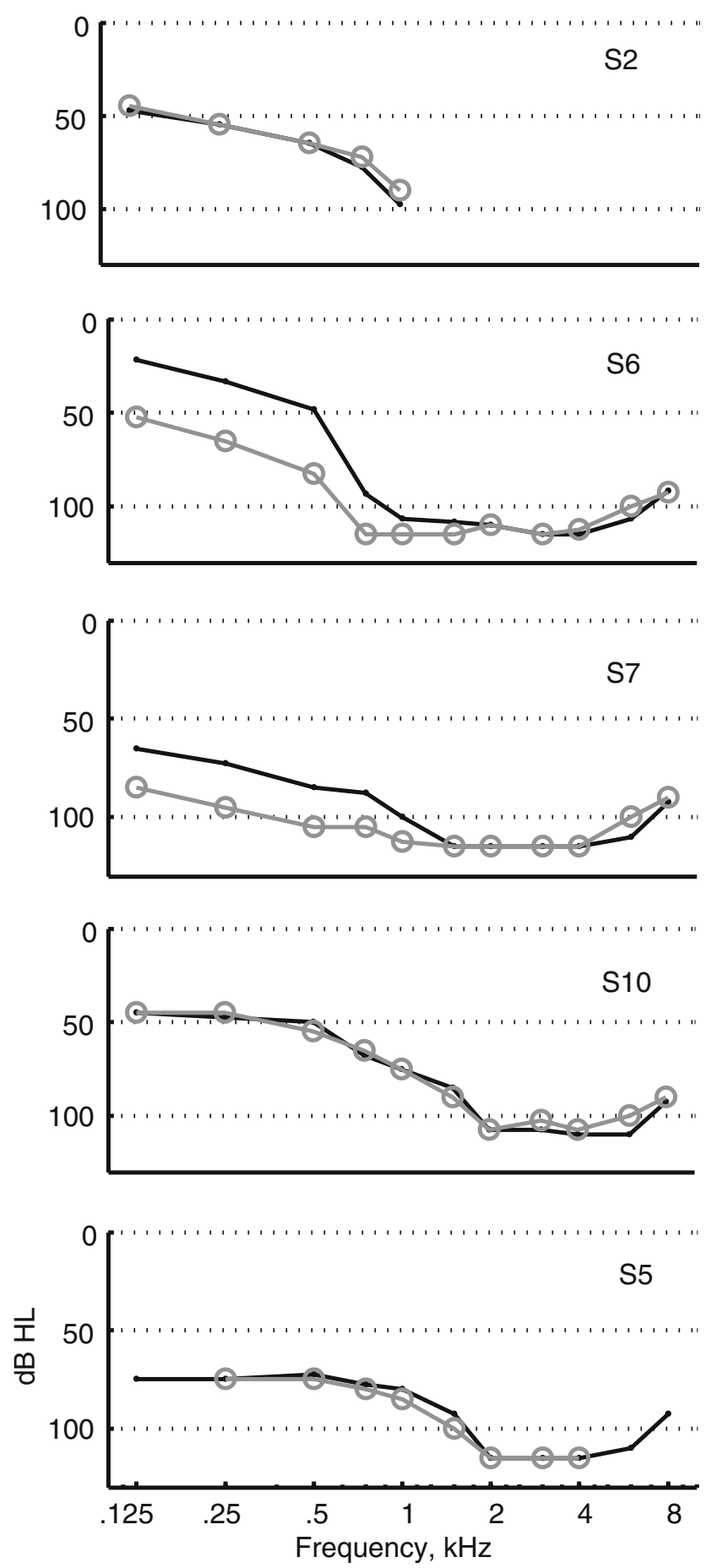

Contralateral
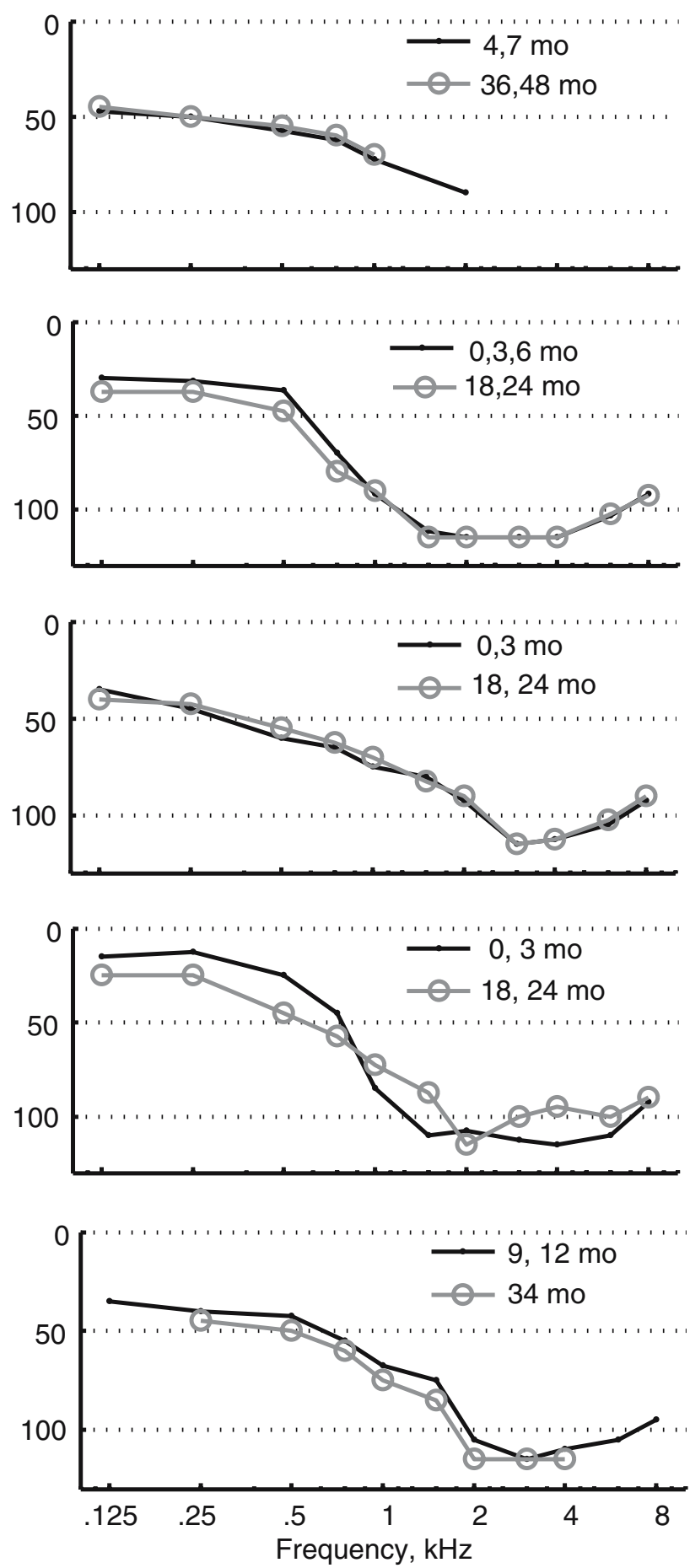

FIG. 3. Early and late audiograms of the same subjects shown in Figure 2. Ipsilateral (implant ear) and contralateral (non-implant ear) audiograms are shown in the left and right columns, respectively. Black lines indicate early audiograms and gray lines with circles indicate late audiograms. Each audiogram (except for S5) is averaged over at least two measurements for each subject and time period. S2: early= (4, 7 months), late $=(36,48$ months); S6: early=(0, 3, 6 months), late=(18, 24 months); S7: early=(0, 3 months), late $=(18,24$ months $) ; S 10$ : early $=(0,3$ months $)$, late=(18, 24 months $) ; S 5:$ early=(9, 12 months $)$, late=(34 months).

understanding was not affected significantly by the change in perceived pitch and also supporting the assumption that the status of the peripheral auditory system remained stable throughout the time course of the measurements. For the one subject S10 whose speech scores were initially poor (at the level of chance), scores were found to improve at later testing around 28 months. The likely reason for the abnor- 

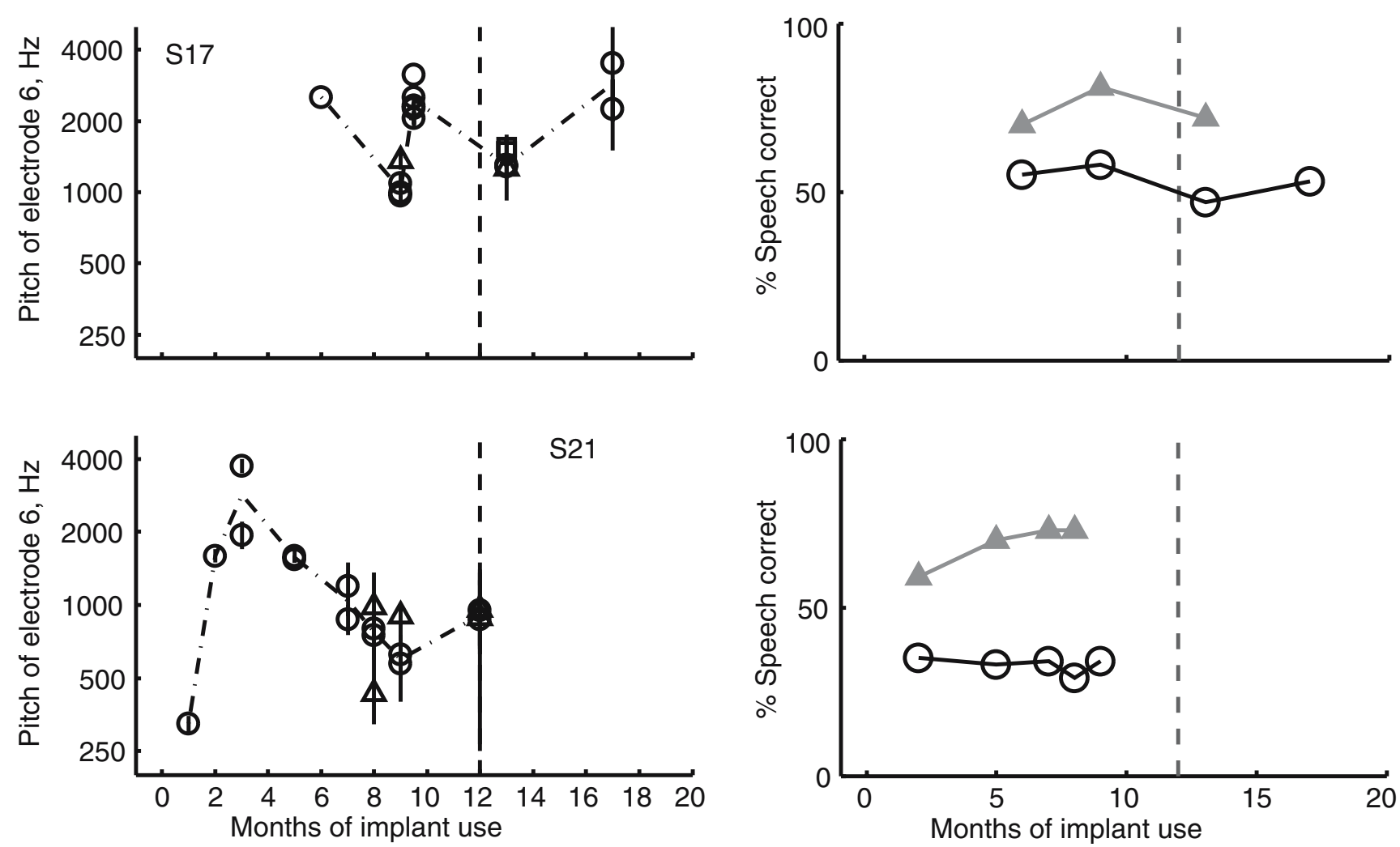

FIG. 4. Pitch sensations and speech performance of two subjects showing atypical, nonsystematic changes in pitch over time. Plotted as in Figure 2. Left column: S17 had highly variable pitch matches even within a single session, suggesting an inability to reliably match pitch. S21 exhibited a systematic rise in pitch up to 3 months, then a drop in pitch subsequently which appears stabilized at 7-8 months. Right column: As with the other subjects, speech scores either did not change or improved slightly over the same time period of the pitch change.

mally low speech score at 12 months was that this speech score was derived with the experimental SPEAR processor (patient was using both SPEAR and Nucleus 24 processors at the time, but "hated" the SPEAR processor); patient performed much better when tested with the Nucleus 24 processor at 28 months.

Figure 3 shows that the subjects' residual hearing did not change significantly over time, especially in the contralateral (non-implanted) ear. Audiograms in the ipsilateral (implanted) ear changed by $20-30 \mathrm{~dB}$ in two out of five subjects (Fig. 3, left column), while audiograms in the contralateral (non-implanted ear) did not change significantly over time for all five subjects (Fig. 3, right column). The important aspect of this figure is that the contralateral hearing used as the acoustic reference for electric pitch matches did not change. Therefore, a change in contralateral hearing thresholds, known to cause diplacusis (Albers and Wilson 1968), does not explain the changes in pitch sensation over time.

It is also highly unlikely that these pitch changes are due to peripheral changes in electrode position or cochlear structure as a result of implantation trauma because electrode impedances, threshold and comfort electrical stimulation levels, and electrically evoked compound action potentials all showed little or no change in these subjects after the first few months of implantation up to at least 1 year of implant use (not shown); this is typical of adult cochlear implant patients, in general, where these parameters are constant for at least 2 years (Hughes et al. 2001).

Additional data are shown for seven other subjects at shorter time scales in Figures 4 and 5; again, the entire pitch-matched bracket (range of frequencies considered "similar") for each data point is also shown as vertical lines around each data point.

The two subjects in Figure 4 exhibited atypical, nonsystematic changes in pitch sensation over time. Subject S17 had highly variably pitch matches, even within a single day. Subject S21, on the other hand, exhibited an interesting and systematic rise in pitch from 300 to as high as $4,000 \mathrm{~Hz}$ over hookup to 3 months, then a systematic fall in pitch from 3 to $7-$ 8 months, after which the pitch seemed to stabilize. However, while the pitch changed nonsystematically over time for these subjects, the speech scores showed either no change or gradual improvements, as for the subjects in Figure 2. 

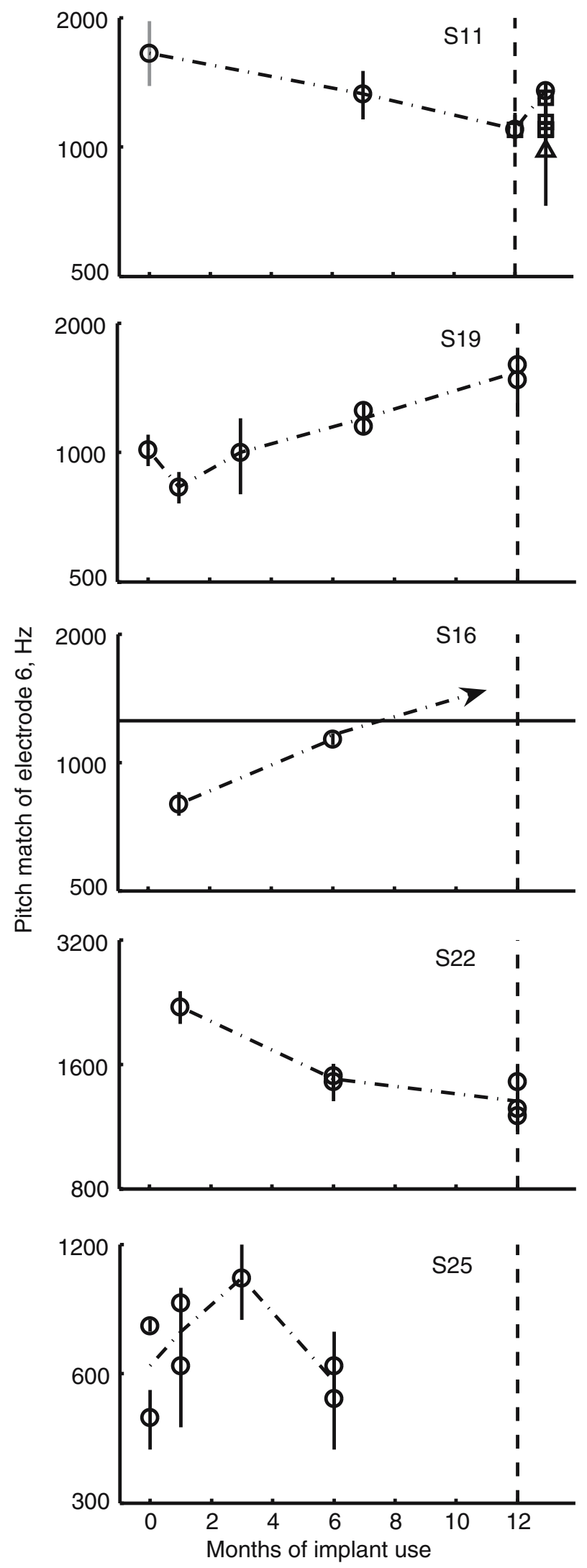

The remaining five subjects appeared to have pitch functions that changed systematically and gradually over time, if at all, as shown in Figure 5. Specifically, two out of five of these subjects (S11 and S22) showed a decrease in pitch over time, while two out of five subjects (S19 and S16) showed an increase in pitch over time. The remaining subject, S25, had broader pitch brackets and within-session variability than the others, and it remains to be seen whether there will be any significant changes over time. Interestingly, the two subjects whose pitch dropped over time in this group also had the two highest early pitch matches of this group, at around 2,000 Hz, compared with $500-1,000 \mathrm{~Hz}$ for the subjects whose pitch increased over time.

It should be noted that one of these subjects (S11) did perceive two tones from implant stimulation of a single electrode, but these two tones were not far apart in frequency, and this subject also occasionally perceived two tones in the non-implant ear as well (monaural diplacusis).

There is a smaller amount of data for the other, more basal electrodes, mainly because these electrodes tend to elicit a higher pitch sensation that is often increasingly more difficult to match to an audible acoustic frequency due to the high-frequency hearing loss in the contralateral ear. These data are shown in Figure 6 for four subjects (S11, S10, S19, and S21); note that unlike Figures 2, 4, and 5, the pitch-matched ranges of each data point are not shown here for clarity, but are usually of a similar range, i.e., one-half- to one-octave wide. The results for these electrodes are mixed; it is not yet clear whether pitch sensations for these electrodes follow the same trends as seen for electrode 6 .

We also measured the effects of current level and pulse rate on pitch sensations in one subject (S21 at 9 months). Figure 7 shows the results obtained using the manual (circles) and randomized (triangles) methods. Clearly, current level does not change the pitch match range significantly for either method (Fig. 7a). While pitch ranges were slightly higher overall for the randomized method in this case, this was not a consistent effect, as the bottom row of Figure 4 shows that in this same subject, the randomized method could yield both lower or higher pitches than the manual method at 8 months or identical pitches at 12 months. These differences due

FIG. 5. Pitch sensations of five other subjects over shorter time scales $(<1$ year). Plotted as in the left column of Figure 2. Slow changes suggest that long-term pitch changes seen in Figure 2 may be gradual. The last data point for S16 is marked with an arrow to indicate that an acoustic pitch match could not be found at the latest time point (the electric pitch sensation was higher than the audible frequency range of this subject, indicated by thin horizontal line). 

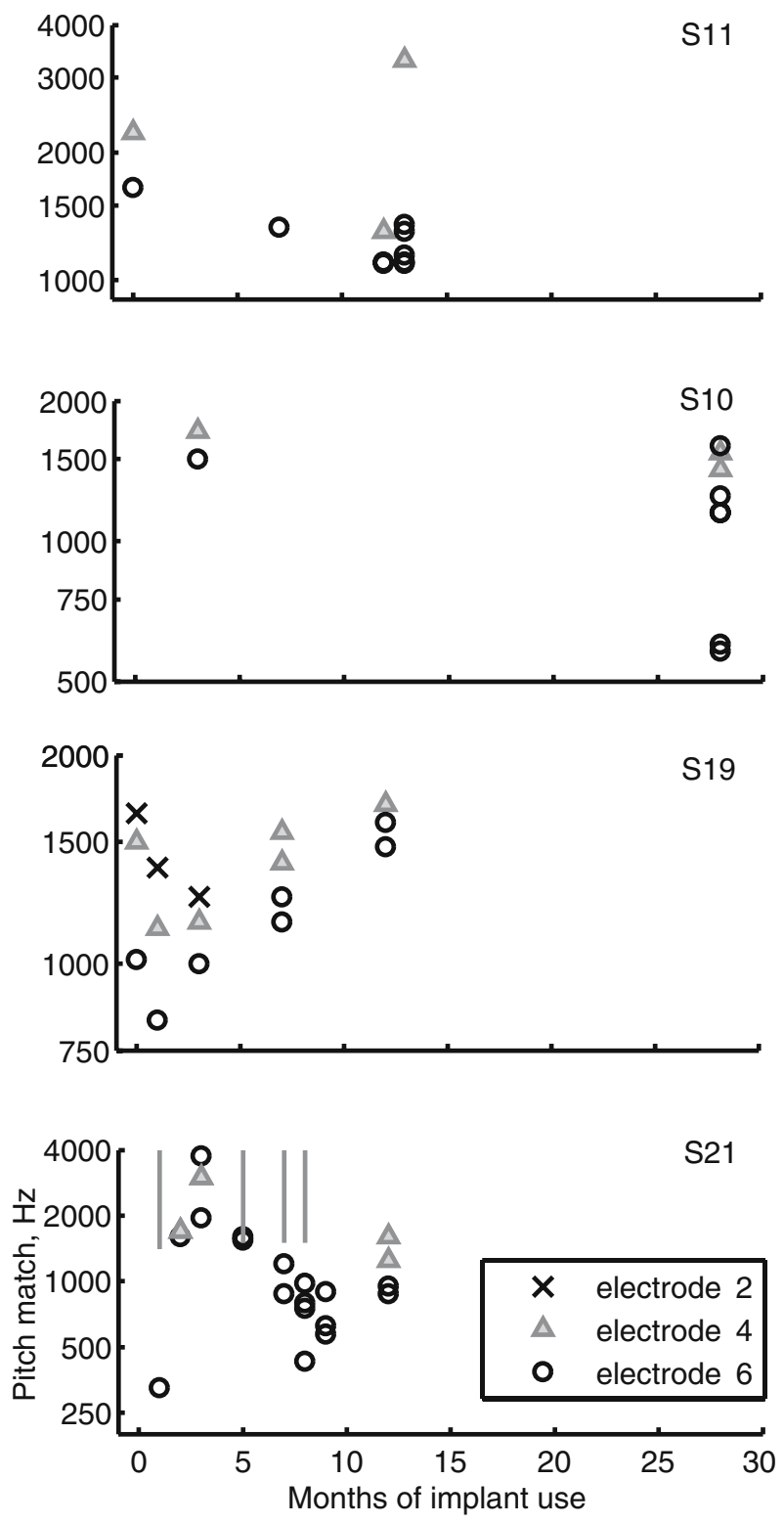

FIG. 6. Pitch sensations across different electrodes over time for four subjects. Plotted as in right column of Figure 2; however, note that pitch match brackets are not shown here for clarity. Different symbols indicate different electrodes, not pitch matching method (all manual in this figure). Electrode 4 (gray triangles) and electrode 2 (black $x$ symbols) are shown together with electrode 6 (open black circles). Gray vertical lines for S21 indicate the lower bound of electrode 4 up to the end of the audible range (subject could not match an upper bound).

to method were also small compared to the changes over time in this subject (compare to 1 and 3 months results, gray triangles/lines). Similarly, changing the pulse rate from 1,200 to 1,800 pps yielded a similar pitch matched range (randomized method; Fig. 7b).

As noted earlier for Figures 2 and 5, the subjects whose pitch dropped over time also had the highest early pitch matches. This suggests that changes in pitch over time are driven by experience with the implant, such as the speech frequencies mapped to the processor. These speech processor frequencies can differ markedly from the predicted Greenwood frequencies for the hybrid electrode insertion depth, and this difference may be a driving force for changes in perceived pitch. Figures 8 compares early and late

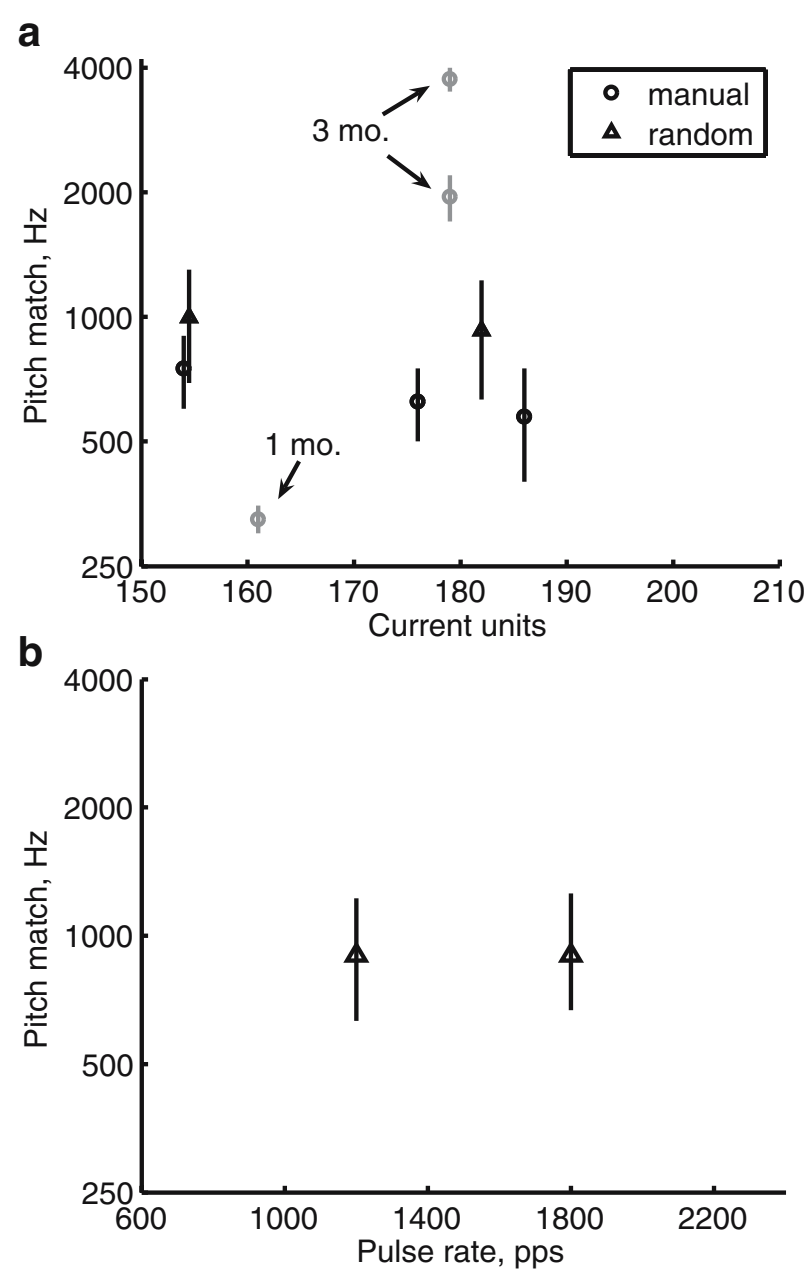

FIG. 7. Effects of varying current level and pulse rate on pitch sensations for a single subject (S21) at 9 months (heavy black symbo/s). (a) Effects of current level. All pitch matches were obtained at 1,200 pps. As in Figure 2, the vertical lines around the circles indicate the range of acoustic frequencies considered similar to the electric pitch, not the variance of the estimate. The vertical lines around the randomly presented computer pitch matches (triangles) indicate the $25-75 \%$ points of the psychometric function. While the computer pitch matches in this case differ slightly from the manual matches here, the difference is small compared to the changes observed over time, e.g., compared to the lowest pitch match at 1 month, and the highest pitch matches at 3 months (open gray circles). Furthermore, there is no effect of changing current levels between 155 and 185 current units (qualitatively associated with medium to loud sound levels). (b) Effects of pulse rate. Pitch matches were obtained similar current levels for 1,200 pps (182 c.u.) and 1,800 pps (178 c.u.). There was no significant difference in the pitch-matched ranges at these two different pulse rates. 


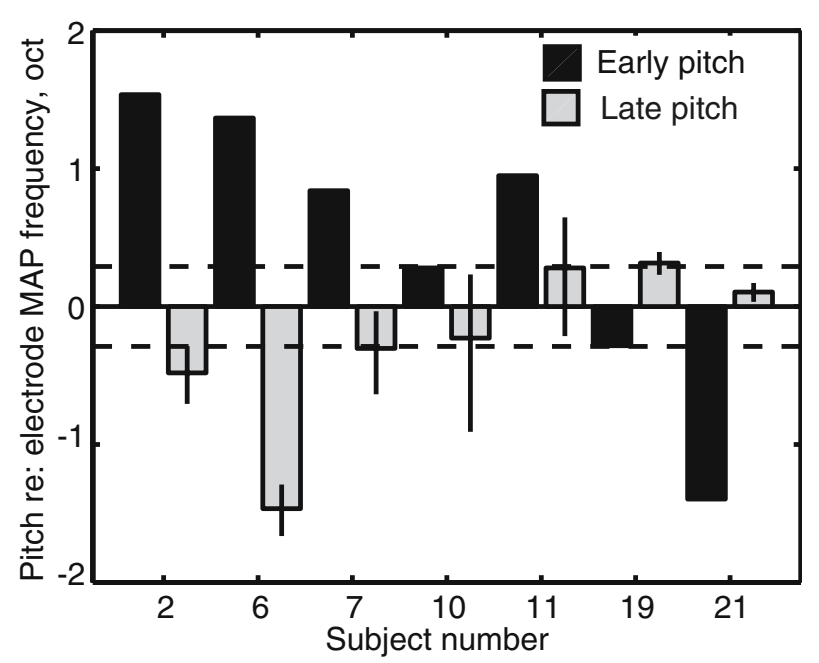

FIG. 8. Early and late pitch matches relative to the center of the preferred processor MAP frequency band for electrode 6. Standard deviations are indicated by vertical lines when multiple pitch matches were obtained. A value near 0 indicates that the pitch match was close to the band center frequency. The horizontal dotted lines show the average range of the MAP frequency band. Only subjects with both early and late pitch match data are shown; subject S17 was excluded because of high variability. electrode six-pitch matches for seven subjects with long-term results (S2, S6, S7, S10, S11, S19, and S21) relative to their preferred, or most often used, speech processor frequency ranges allocated to electrode 6 . The $y$-axis is the pitch sensation relative to the logarithmic center of the frequency range, where 0 reflects a perfect match to the center, and the dotted lines indicate the relation to the average upper and lower bound of the frequency allocation of the speech processor for electrode 6 . The vertical lines indicate standard deviations at times when multiple estimates were taken. The early pitch matches (black bars) are clearly more mismatched to the frequency allocation range (outside the dotted lines) than the late pitch matches (gray bars) for four out of seven subjects (S2, S7, S11, S21). For two out of seven subjects (S10 and S19), the early pitch match is already within the range of frequency allocation, and the late pitch match remains within the range, suggesting a lack of driving force for change. The last subject, S6, shows an overshoot effect in going from a too high pitch to a too low pitch for the allocated frequency range. This may mean that other factors besides speech processor frequencies may influence changes in pitch perception. a

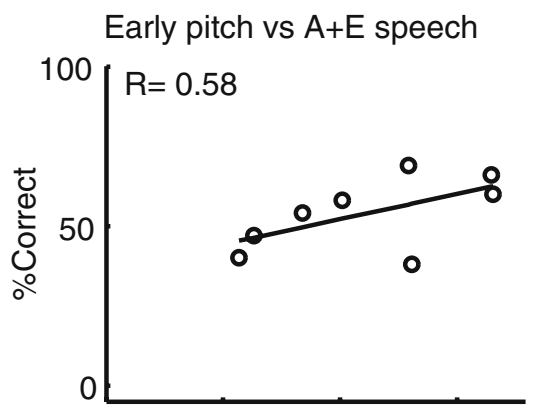

b

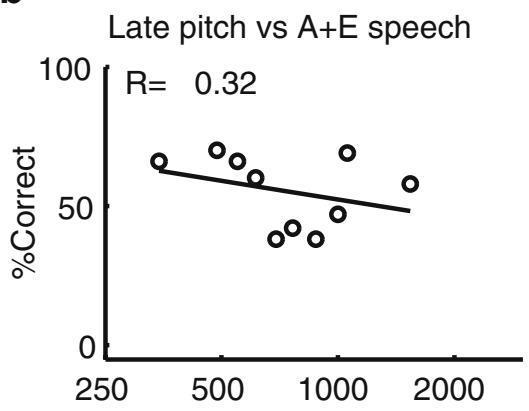

C

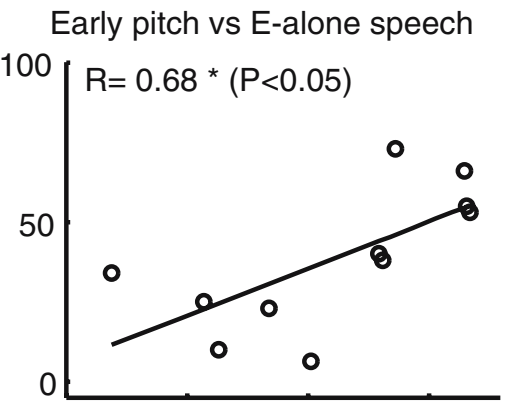

d

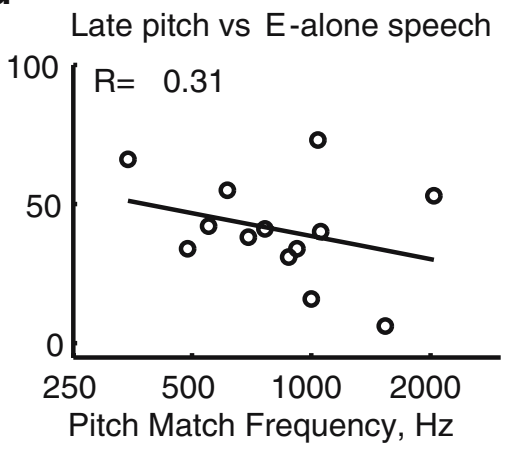

e

Early pitch vs $A$-alone speech

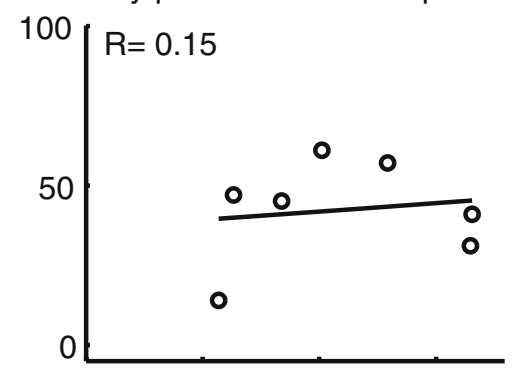

f

Late pitch vs A -alone speech

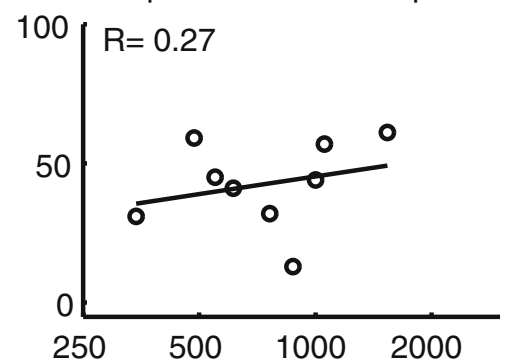

FIG. 9. Long-term speech scores plotted as a function of early- and late-pitch sensations across subjects. Speech reception scores (for consonants) were significantly correlated (Pearson $R$ two-tailed test) with early pitch sensations for the electric-alone $(\mathrm{E}$-alone) $(\mathbf{c} ; P<0.05)$ conditions, but not for the acoustic + electric $(A+E)$ or acoustic-alone (A-alone) condition (a and e); in contrast, for late pitch sensations, no significant correlations were observed $(\mathbf{b}, \mathbf{d}, \mathbf{f})$. When speech scores are correlated vs linear instead of log frequency, the results are the same, with all conditions remaining uncorrelated except E-alone, which is even more significantly correlated $(R=0.75, P<0.01)$. Early subjects include: S2, S6, S7, S9, S10, S11, S14, S16, S17, S19, and S21. Late subjects include: S2, S3, S5, S6, S7, S8, S10, S11, S12, S15, S17, S19, and S21. 

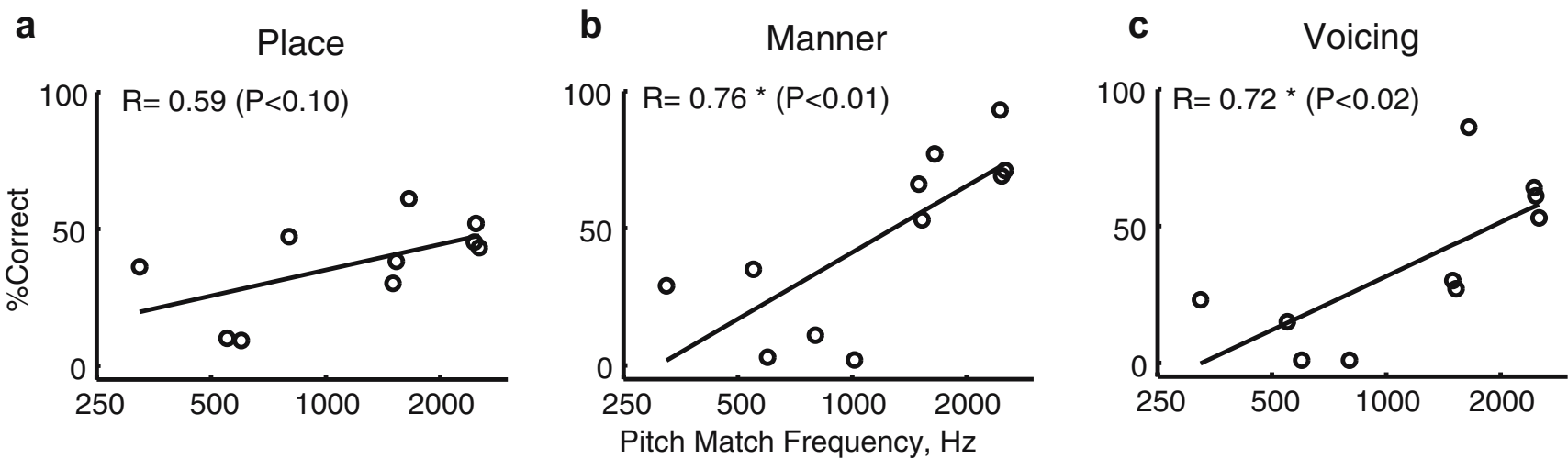

FIG. 10. Electric-alone speech recognition of consonants broken down by place, manner, and voicing cues, plotted as a function of early pitch sensations across subjects. Speech scores were correlated with early pitch sensations for all cues (Pearson $R$ two-tailed test), but most significantly for manner and voicing: place $(\mathbf{a} ; P<0.10)$, manner $(\mathbf{b} ; P<0.01)$, and voicing $(\mathbf{c} ; P<0.02)$.

\section{Correlations of electric pitch with speech} reception performance

We also compared pitch sensations with long-term speech reception performance with the implant. Figure 9 shows that early, but not late, pitch sensations were correlated with speech reception performance in hybrid subjects under electric-only conditions. Specifically, speech performance under electric-only conditions was significantly and positively correlated with early pitch sensation (Fig. 9c), with subjects with high early pitch sensations performing better with the implant than those with low pitch sensations. These results are consistent with our previously stated hypothesis that higher early pitch sensations are indicative of a larger population of surviving neurons located near the hybrid "short" electrode implant in the basal end of the cochlea. No significant correlation was observed for the acoustic + electric or acoustic-alone condition, which measured performance independent of the implant (Fig. 9a,e). Note that acoustic + electric and acoustic-alone conditions have fewer data points due to the fact that while direct electric data were collected for all subjects, there was not always enough time to also get data for acoustic + electric or acoustic-alone.

Also note that early and late pitch matches were sometimes obtained for different subject populations either because of limited access to some patients (from other clinical trial centers) or because we were sometimes initially unable to match early high pitch sensations to an audible frequency, but were able to match later pitch sensations to a lower frequency in the audible frequency range (see below).

In contrast, late pitch sensations did not predict speech performance; no correlation of speech with late pitch matches was observed under any condition (Fig. 9b,d,f). This latter result suggests that late pitch sensations are determined less by peripheral factors and more by central influences unrelated to speech perception.

Electric-alone speech recognition performance was also broken down by place, manner, and voicing cues (Miller and Nicely 1955). As with the total speech scores, Figure 10 shows that these scores were significantly correlated with early pitch sensations for all three cues. These scores were again not significantly correlated with late pitch sensations for any type of cue (not shown).

\section{DISCUSSION}

\section{Pitch changes over time}

The data shown here suggest that pitch perceived through a hybrid cochlear implant can change over time. In particular, two patients, S2 and S6, exhibited a large downward shift, of more than two octaves, in pitch sensation for the most apical electrode over 3-5 years of implant experience (Fig. 2). Other patients, S7 and S10, had smaller pitch shifts over 2-3 years. However, because of the infrequent sampling of pitch estimates over time, it is unclear whether these changes were gradual or sudden or how much of the changes could be attributed to intra- and intersession variability.

A second group of patients was tested at shorter time intervals and with more repeat measurements, up to 13 months (Figs. 4, 5). Several of these patients also showed small changes in pitch sensation over this short time frame. The preliminary data for these subjects suggest systematic and slow changes that could eventually lead to the larger changes observed for S2 and S6 at long time scales and imply that the changes seen for the former group are also systematic and slow. However, intra- and intersession variability on the order of one-half to one octave is also evident 
at the shorter time intervals for these subjects, especially S17 and S21. While subjects S2 and S6 showed pitch changes much larger than those attributable to variability, it is not known whether the majority of subjects will eventually show significant changes larger than those attributable to variability. Regardless, the finding of highly variable pitch estimates in some patients within and across sessions is still relevant. These data emphasize the importance of checking the contribution of variability to pitch estimates, a factor that has been perhaps under-emphasized in previous studies of cochlear implant pitch perception.

Why are the pitch estimates so variable in the hybrid patients? As with long-electrode patients, sometimes patients report that the quality of sounds from individual electrodes are less precise than the acoustically presented tones coming from a headphone. This suggests that for these patients, electrical stimulation may produce a more diffuse than puretone-like percept. It is also possible that some patients are pitch-matching to multiple tonal percepts from the implant and that this accounts for some of the variability.

Alternatively, some patients may have octave confusions during the pitch-matching task, and these confusions may contribute to the variability or changes seen in the data over time. If so, then pitch rankings should be changing non-monotonically from "lower" to "higher" and back with increased acoustic frequency, and similarly at approximately each octave. Two subjects (S11 and S21) exhibited possible octave confusions at their latest time points, which could plausibly account for the variability seen in their pitch matches. Note that these octave confusions encompassed only one octave, i.e., the effect did not persist beyond one octave of the frequency range. Furthermore, this effect was not seen in most subjects, even with fine frequency sampling over a wide frequency range. Therefore, these octave confusions are a plausible partial explanation for pitch changes or variability of up to one octave, but not likely to explain changes of two octaves or more. Nonetheless, as it is difficult to know exactly what patients are perceiving in all cases in the present data and in previous pitch-matching studies, some caution is appropriate.

Long-term changes in pitch perception associated with a fixed place of stimulation in the auditory periphery have not been reported previously. These results suggest that under "unnatural" stimulation conditions, the central auditory system might have a major contribution to the tonotopic coding of sound frequency. In fact, this would not be surprising given the presence of analogous perceptual changes in other sensory systems. The cerebral cortex is known to reorganize greatly in response to major, traumatic changes in afferent input, such as digit amputation or cochlear lesions (Buonamano and Merzenich 1998). Sensory systems have also been shown to adapt to altered patterns of sensory input. In the visual system, for instance, it has been shown in owls that while prisms will initially cause systematic localization errors, the owls eventually adapt their visuo-motor responses and even adjust their auditory maps to align with the new visual maps; furthermore, these changes are reflected physiologically (review: Knudsen 2002).

\section{Relationship of electric pitch to cochlear place}

The most apical electrode in the hybrid implant is implanted approximately $10.5 \mathrm{~mm}$ from the cochlear base. If this electrode is stimulating nearby dendritic nerve fibers associated with the hair cells, the Greenwood frequency-place function predicts a pitch sensation between 2,800 and $4,700 \mathrm{~Hz}$, depending on electrode insertion angle and cochlear length (Greenwood 1990; Leake et al. 2006). Stimulation of spiral ganglion cells instead of dendrites, on the other hand, would have little effect on predicted pitch sensation in the basal region of the cochlea under consideration (Leake et al. 2006). Almost all of the pitch sensations are lower than predicted, by place of electrical stimulation, consistent with most previous studies of pitch sensation in cochlear implants. Often proposed reasons for lower-thanexpected pitch estimates include reduced basal nerve survival, nonlinear stimulation patterns, and stimulation of spiral ganglion cells instead of dendrites (although the last seems to be a small factor in the basal region as discussed by Leake et al. 2006).

The results of the current study suggest another explanation. Several subjects with both early and late pitch data had a downward shift in pitch sensation over time (Fig. 8). The speech perception data show that early, but not late, pitch sensations were strongly correlated with speech reception performance in hybrid subjects (Fig. 9). These two results together suggest that while often lower than expected, early pitch sensations may still reflect electrical stimulation patterns along the tonotopic organization of the nerve array. On the other hand, late pitch sensations may be determined less by peripheral factors and more by central influences.

Shifts in pitch sensation over time may help to explain some discrepancies in previous pitch estimation studies. Three of the four previous studies of absolute implant pitch perception have yielded results inconsistent with the cochlear place principle, with pitch sensations one to three octaves lower than expected based on cochlear place of stimulation (Dorman et al. 1994; Blamey et al. 1996b; Boex et 
al. 2006). The one study that found pitch sensations to correspond roughly to cochlear place frequency differed from the others in that it was conducted in a unilaterally deaf patient during (and soon after) implantation surgery (Eddington et al. 1978). Thus, the Eddington et al. data are similar to the "Early" pitch matches of the present study, while the other studies cited above correspond to "Late" data. While it is known that hearing loss can cause diplacusis, i.e., a difference in the pitch sensation between the two ears, these differences are typically on the order of $12-25 \%$ in unilaterally hearing impaired subjects (Gaeth and Norris 1965; Knight 2004) and much smaller in binaurally impaired subjects (Robinson and Gaeth 1975; Burns and Turner 1986). Such small differences due to diplacusis clearly cannot account for a one- to three-octave discrepancy in pitch sensation. Experience-dependent changes in pitch sensation over time may better explain the large discrepancies between these studies, as the other studies with low pitch matches were conducted long after implantation, usually years later.

\section{Mechanisms of electric pitch shifts}

Previously, only modest plasticity in the form of sharpened or enhanced tuning due to behavioral conditioning has been observed in normal-hearing listeners and expanded receptive fields due to cochlear lesions in animal models (review: Irvine and Wright 2005). Shifts in absolute pitch perception over time, on the other hand, have not been reported other than in diplacusis with sudden hearing loss, which is usually attributed to mechanical/peripheral causes and of a much smaller scale than the maximum of approximately two octaves observed in the present study.

What could be the driving force for shifts in absolute pitch perception? One possible driving force is suggested by the convergence of the late pitch sensations to the relatively low speech processor frequencies allocated to the corresponding electrode, as shown in Figure 8. This mismatched frequency allocation will cause perceived speech to sound very different from the original, real-world speech representation before implantation and may cause the brain to adapt or remap the frequencies it hears through the implant so that speech "sounds more like it is supposed to". While the frequency allocations are not exactly matched to the new pitch sensations for individual subjects, it is possible that the brain shifts the sensation only approximately to restore the "normal" quality of speech. The simultaneous acoustic stimulation from the residual acoustic hearing in the opposite ear may also be a driving force in auditory plasticity. In other words, the pitch shifts may be driven by the difference in perceived pitch between the two ears due to the severe spectral shift from the implant, i.e., implant-induced diplacusis much larger than that seen in normal hearing or hearing-impaired subjects may be a driving force for perceptual remapping of frequency. Note that while one of the assumptions is that the acoustic reference, or opposite ear, is stable, it is certainly also possible that the central perception of the acoustic reference could be increasing over time. Alternatively, the pitch shifts may even be driven by perceived differences between the acoustic and electric components of a sound heard in the same ear.

Another possibility is that decreases in the number of surviving high frequency nerve fibers in the implanted ear would cause the implant to stimulate lower frequency fibers instead, thus, eliciting a lower pitch sensation. This seems unlikely because (1) residual hearing remained stable in the ipsilateral ear, within 10-20 dB, in all but two subjects (S6 and S7), and one of these subjects still had considerable usable residual hearing (S6); and (2) electric-only implant speech performance remained the same or even improved slightly over this time period (Figs. 2 and 4).

It is conceivable that traditional long electrode implant patients could also have changes in pitch perception over time. Both hybrid and traditional implant patients note that speech through their implant initially sounds "squeaky", but later sounds more normal and like regular speech [Dorman et al. 1997; personal observations]. This again suggests that implant processors commonly assign speech frequencies to the wrong frequency-place locations, although the mismatch would be much smaller for traditional implant patients. This spectral mismatch appears to have little impact on future speech reception performance, suggesting that implant users can eventually adapt. This belies other short-term studies of speech reception performance with spectrally mismatched speech in normal hearing simulations (Dorman et al. 1997; Fu and Shannon 1999b; Faulkner et al. 2003), but is consistent with longerterm studies showing that cochlear implant users can adapt over months (Rosen et al. 1999; Fu et al. 2005).

\section{Correlations with speech reception and nerve survival}

Previous studies of other factors indirectly related to neuronal survival have generally found that the duration of deafness, but not the cause of deafness or age of onset, are correlated with speech perception in traditional implant subjects (e.g., Kileny et al. 1991; Gantz et al. 1993; Summerfield and Marshall 1995; Rubinstein et al. 1999; for a complete review, see Blamey et al. 1996a). This study suggests that 
absolute pitch perception may also be indirectly correlated with nerve survival. Specifically, individual differences in the amount of nerve survival in the cochlear base is one factor that may explain the highly significant positive correlation of early pitch sensations with electric-only speech reception performance across hybrid implant subjects. In other words, those subjects with greater basal nerve survival in the location of the implant are hypothesized to have both high (early) pitch sensations (corresponding to predictions based on electrode cochlear location) and better speech reception performance; hence, the positive correlation.

This result is in contrast to previous direct measures of nerve survival in human temporal bones of cochlear implant recipients which have shown little or negative correlation of nerve fiber survival with speech performance (Nadol et al. 2001; Khan et al. 2005). The previous lack of correlation may be due to the greater heterogeneity of the traditional implant recipient groups included in these previous studies; these recipients not only have different devices and processing strategies, but also most likely include both pre- and post-lingually deafened individuals. In contrast, the individuals in the current study are highly homogeneous in their use of a single processor type (most used Cochlear brand Nucleus 24 or Freedom, or Cochlear's experimental SPEAR processor which was programmed with similar parameters), processing strategy (ACE), post-lingual onset of deafness, and a highly localized location $(6 \mathrm{~mm}$ in the basal end only) for electrode stimulation. The post-lingual onset of deafness may be especially important for eliminating variance due to cognitive factors. However, future direct verification through temporal bone studies of hybrid implant recipients is needed to verify the hypothesized link of these two parameters to nerve survival.

The results seen here may also seem surprising given previous literature showing that deeper insertion produces lower pitch and higher speech reception performance. The difference between this study and those studies is that insertion depth is considered unlikely to be the main variable that determines pitch sensation in this study. The hybrid implant involves a relatively straightforward insertion with a better repeatability of insertion depth than the long electrode. We consider it more likely that the dominant variable is nerve survival at that particular insertion range near the stimulating electrode, i.e., a low pitch would reflect poor nerve survival nearby and thus poor electrode specificity.

This result may have a clinical application, whatever the reason for the link between pitch sensations and speech performance. The correlation suggests that early pitch sensations can be used as a predictive and diagnostic measure early in hybrid implant use (within 3 months) for whether the patient will do well with the device after a year. These findings may also have important implications for the possible success of binaural cochlear implantation. The observation that pitch can change over time, together with the implication that early pitch matches might more closely reflect peripheral innervation patterns, also suggest that efforts to provide "place-matched" binaural stimulation for patients with two implants might be more closely achieved by using early pitch matches to align frequency mappings to the two ears.

\section{CONCLUSION}

Until now, it has been generally assumed that the periphery dominates sensory perception through the implant and that many aspects of implant performance measured psychophysically (i.e., pitch, frequency resolution, intensity discrimination) reflect peripheral status. If the central auditory system can also influence electric sensations as suggested by these data, then the results of such previous studies may need to be reinterpreted, especially those measuring pitch perception in cochlear implant users. The possibility that absolute pitch sensation can be influenced by experience also has implications for current theories of pitch perception, including the importance of other factors besides cochlear place in the coding scheme (van den Brink et al. 1976).

\section{ACKNOWLEDGEMENTS}

We thank Jessica Taylor and Anna Yao for help with experiments and data collection, Arik Wald and David Wood for programming and technical advice, Mary Lowder, Ann Perreau, and Beth MacPherson for patient MAPs and audiograms, Marla Ross and Jill Buckingham for scheduling subjects, and Aaron Parkinson and Colin Irwin of Cochlear Corporation for providing implant equipment and programming tools. We also thank Paul Abbas, Steven Green, Robert Hong, and the Iowa Auditory Journal Group for comments on the manuscript. Funding for this research was provided in part by research grants RO1DC000377 and 2P50 DC00242 from National Institutes on Deafness and Other Communicative Disorders, National Institutes of Health, and grant RR00059 from the General Clinical Research Centers, NCRR, National Institutes of Health.

\section{REFERENCES}

Albers GD, Wilson WH. Diplacusis. I. Historical review. Arch. Otolaryngol. 87(6):601-603, 1968. 
Baskent D, Shannon RV. Frequency-place compression and expansion in cochlear implant listeners. J. Acoust. Soc. Am. 116(5):3130-3140, 2004.

Blamey PJ, Arndt P, Bergeron F, Bredberg G, Brimacombe J, Facer G, Larky J, Lindstrom B, Nedzelski J, Peterson A, Shipp D, Staller S, WHITFORD L. Factors affecting auditory performance of postlinguistically deaf adults using cochlear implants. Audiol. Neuro-otol. 1(5):293-306, 1996a.

Blamey PJ, Dooley GJ, Parisi ES, Clark GM. Pitch comparisons of acoustically and electrically evoked auditory sensations. Hear. Res. 99(1-2):139-150, 1996b.

Boex C, Baud L, Cosendai G, Sigrist A, Kos Mi, Pelizzone M. Acoustic to electric pitch comparisons in cochlear implant subjects with residual hearing. J. Assoc. Res. Otolaryngol. $7(2): 110-124,2006$.

Buonamano DV, Merzenich MM. Cortical plasticity: from synapse to maps. Annu. Rev. Neurosci. 21:149-186, 1998.

Burns EM, Turner CW. Pure-tone pitch anomalies. II. Pitchintensity effects and diplacusis in impaired ears. J. Acoust. Soc. Am. 79:1530-1540, 1986.

Dorman MF, Smith M, Smith L, Parkin JL. The pitch of electrically presented sinusoids. J. Acoust. Soc. Am. 95(3):1677-1679, 1994.

Dorman MF, Loizou PC, Rainey D. Simulating the effect of cochlear implant electrode insertion depth on speech understanding. J. Acoust. Soc. Am. 102(5):2993-2996, 1997.

Eddington DK, Dobelle WH, Brackmann DE, Mladejovsky MG, PARkIN JL. Auditory prostheses research with multiple channel intracochlear stimulation in man. Ann. Otol. Rhinol. Laryngol. 87(6 Pt 2):1-39, 1978.

Faulkner A, Rosen S, Stanton D. Simulations of tonotopically mapped speech processors for cochlear implant electrodes varying in insertion depth. J. Acoust. Soc. Am. 113(2):10731080, 2003.

Fu QJ, Shannon RV. Effects of electrode configuration and frequency allocation on vowel recognition with the Nucleus-22 cochlear implant. Ear Hear. 20(4):332-344, 1999a.

Fu QJ, Shannon RV. Recognition of spectrally degraded and frequency-shifted vowels in acoustic and electric hearing. J. Acoust. Soc. Am. 105(3):1889-1900, 1999b.

Fu QJ, Nogaki G, Galvin JJ 3RD. Auditory training with spectrally shifted speech: implications for cochlear implant patient auditory rehabilitation. J. Assoc. Res. Otolaryngol. 6(2):180189,2005

GaEth JH, Norris TW. Diplacusis in unilateral high frequency hearing losses. J. Speech Hear. Res. 8:63-75, 1965

Gantz BJ, Turner CW. Combining acoustic and electric hearing. Laryngoscope. 113:1726-1730, 2003.

Gantz BJ, Woodworth GG, Knutson JF, Abbas PJ, Tyler RS. Multivariate predictors of audiological success with multichannel cochlear implants. Ann. Otol. Rhinol. Laryngol. 102:909916, 1993

GREENWOOD D. A cochlear frequency-position function for several species-29 years later. J. Acoust. Soc. Am. 87(6):2592-2605, 1990.

Hughes ML, Vander Werff KR, Brown CJ, Abbas PJ, Kelsay DMr, Teagle HFB, Lowder MW. A longitudinal study of electrode impedance, the electrically evoked compound action potential, and behavioral measures in nucleus 24 cochlear implant users. Ear Hear. 22(6):471-486, 2001.
Irvine DRF, Wright BA. Plasticity of spectral processing. In: Malmierca MS and Irvine DRF (eds) Auditory Spectral Processing Vol. 70. London, Elsevier Academic Press, pp. 435-472, 2005.

James C, Blamey P, Shallop JK, Incerti PV, Nicholas AM. Contralateral masking in cochlear implant users with residual hearing in the non-implanted ear. Audiol. Neurootol. 6:87-97, 2001.

Khan A, Whiten DM, Nadol JB, Eddington DK. Histopathology of human cochlear implants: correlation of psychophysical and anatomical measures. Hear. Res. 205:83-93, 2005.

Kileny PR, Zimmerman-Phillips S, Kemink JL, Schmaltz SP. Effects of preoperative electrical stimulability and historical factors on performance with multichannel cochlear implant. Ann. Otol. Rhinol. Laryngol. 100:563-568, 1991.

KNight RD. Diplacusis, hearing threshold, and otoacoustic emissions in an episode of sudden, unilateral hearing loss. Int. J. Audiol. 43:45-53, 2004.

KNUDSEN EI. Instructed learning in the auditory localization pathway of the barn owl. Nature 417:322-328, 2002.

Leake PA, Stakhovskaya O, SRidhar D. Estimating represented frequencies for cochlear implant electrodes in human temporal bone and imaging studies. Assoc. Res. Otolaryngol. Abstracts, 2006.

LevitT H. Transformed up-down methods in psychoacoustics. J. Acoust. Soc. Am. 49:467-477, 1971.

McKay CM, McDermott HJ, Carlyon RP. Place and temporal cues in pitch perception: are they truly independent? ARLO 1:25-30, 2000.

Milier G, Nicely P. An analysis of perceptual confusions among some English consonants. J. Acoust. Soc. Am. 27(2):338-352, 1955.

Nadol JB, Shiao JY, Burgess BJ, Ketten DR, Eddington DK, Gantz BJ, Kos I, Montandon P, Coker NJ, Roalnd JT, Shallop JK. Histopathology of cochlear implants in humans. Ann. Otol. Rhinol. Laryngol. 110:883-891, 2001.

Robinson DO, GaETh JH. Diplacusis associated with bilateral high frequency loss. J. Speech Hear. Res. 18:5-16, 1975.

Rosen S, Faulkner A, Wilkinson L. Adaptation by normal listeners to upward spectral shifts of speech: implications for cochlear implants. J. Acoust. Soc. Am. 106:3629-3636, 1999.

Rubinstein JT, Parkinson WS, Tyler RS, Gantz BJ. Residual speech recognition and cochlear implant performance: effects of implantation criteria. Am. J. Otol. 20:445-452, 1999.

SHANNON RV. Multichannel electrical stimulation of the auditory nerve in man. I. Basic psychophysics. Hear. Res. 11:157-189, 1983.

Summerfield AQ Marshall DH. Preoperative predictors of outcomes from cochlear implantation in adults: performance and quality of life. Ann. Otol. Rhinol. Laryngol. Suppl. 166:105-108, 1995.

Tong YC, Clark GM, Blamey PJ, Busby PA, Dowell RC. Psychophysical studies for two multiple-channel cochlear implant patients. J. Acoust. Soc. Am. 71:153-160, 1982.

Townshend B, Cotter N, van Compernolle D, White RL. Pitch perception by cochlear implant subjects. J. Acoust. Soc. Am. 82:106-115, 1987.

Turner CW, Souza PE, Forget LN. Use of temporal envelope cues in speech recognition by normal and hearing-impaired listeners. J. Acoust. Soc. Am. 97:2568-2576, 1995.

Turner CW, Gantz BJ, Vidal C, Behrens A. Speech recognition in noise for cochlear implant listeners: benefits of residual acoustic hearing. J. Acoust. Soc. Am. 115:1729-1735, 2004.

van den Brink G, Sintnicolaas K, van Stam WS. Dichotic pitch fusion. J. Acoust. Soc. Am. 59:1471-1476, 1976. 